\title{
Electron hole-phonon interaction, correlation of structure, and conductivity in single crystal $\mathrm{La}_{0.9} \mathrm{Sr}_{0.1} \mathrm{FeO}_{3-\delta}$
}

\author{
A. Braun, ${ }^{1, a)}$ J. Richter, ${ }^{1}$ A. S. Harvey ${ }^{2}$ S. Erat, ${ }^{1,2}$ A. Infortuna, ${ }^{2}$ A. Frei, ${ }^{3}$ \\ E. Pomjakushina, ${ }^{4,5}$ Bongjin S. Mun, ${ }^{6,4}$ P. Holtappels, ${ }^{1}$ U. Vogt, ${ }^{1}$ K. Conder, ${ }^{4}$ \\ L. J. Gauckler, ${ }^{2}$ and T. Graule ${ }^{1}$ \\ ${ }^{1}$ Laboratory for High Performance Ceramics, Empa-Swiss Federal Laboratories for Materials Testing \\ and Research, $\mathrm{CH}-8600$ Dübendorf, Switzerland \\ ${ }^{2}$ Department for Nonmetallic Inorganic Materials, ETH Zürich-Swiss Federal Institute of Technology, \\ CH-8037 Zürich, Switzerland \\ ${ }^{3}$ Solar Technology Laboratory, Paul Scherrer Institut, CH-5232 Villigen PSI, Switzerland \\ ${ }^{4}$ Laboratory for Development and Methods, Paul Scherrer Institut, CH-5232 Villigen PSI, Switzerland \\ ${ }^{5}$ Laboratory for Neutron Scattering, Paul Scherrer Institut and ETH Zürich, \\ CH-5232 Villigen PSI, Switzerland \\ ${ }^{6}$ Department of Applied Physics, Hanyang University, Ansan, Kyunggi-Do 426-791, Republic of Korea \\ ${ }^{7}$ Advanced Light Source, Lawrence Berkeley National Laboratory, Berkeley, California 94720, USA
}

(Received 20 October 2008; accepted 24 November 2008; published online 30 December 2008)

\begin{abstract}
The conductivity and structure of the hole-doped polaron conductor $\mathrm{La}_{0.9} \mathrm{Sr}_{0.1} \mathrm{FeO}_{3-\delta}$ are reported for elevated temperatures. The conductivity increases exponentially with temperature to a maximum and decreases for $T>700 \mathrm{~K}$ following a power law, accompanied by a shift of spectral weight in the photoemission valence band. The conductivity decrease is accompanied by a phase transformation, due to the reduction of $\mathrm{Fe}$, as evidenced by $\mathrm{x}$-ray absorption spectra. Additional fine structures in the conductivity are correlated with a strong decrease in valence band intensity near $E_{F}$ and with the onset of a corresponding structural transition. () 2008 American Institute of Physics.
\end{abstract}

[DOI: $10.1063 / 1.3049614]$

Properties of strongly correlated fermion systems are rarely studied at high temperatures, although such temperatures are relevant for solid oxide fuel cells and sensors, for example. We report on single crystal $\mathrm{La}_{0.9} \mathrm{Sr}_{0.1} \mathrm{FeO}_{3-\delta}$ (LSF10), which comes close in properties to the insulating parent compound $\mathrm{LaFeO}_{3} \cdot \mathrm{LaFeO}_{3}$ is an antiferromagnetic (AFM) charge transfer insulator with a band gap energy $E_{g}$ of $2 \mathrm{eV}$ and Néel temperature of $750 \mathrm{~K}$ and has orthorhombic symmetry with four distorted pseudocubic cells in the space group Pbnm. The Fe has its spin antiparallel to its six nearest neighboring $\mathrm{Fe}$ atoms, with parasitic ferro- and ferrimagnetism $^{1,2}$ for low Sr doping. Heterovalent substitution of $\mathrm{La}^{3+}$ with $\mathrm{Sr}^{2+}$ (hole doping) creates conducting electron hole states with $\mathrm{O}(2 p)$ character near $E_{F},{ }^{3}$ accompanied by a decrease of $\mathrm{T}_{\mathrm{N}}$. $\mathrm{SrFeO}_{3}$ is an AFM metallic conductor. ${ }^{4}$

$\mathrm{La}$ and $\mathrm{Sr}$ are considered to retain their formal valence $\mathrm{La}^{3+}$ and $\mathrm{Sr}^{2+}$. Charge balance is maintained by adjustment of the valence of the $\mathrm{Fe}$ and by oxygen deficiency $\delta$ in the $\mathrm{La}_{(1-x)} \mathrm{Sr}_{x} \mathrm{FeO}_{3-\delta}$. The formal valences of $\mathrm{Fe}$ in $\mathrm{LaFeO}_{3}$ and $\mathrm{SrFeO}_{3}$ are $\mathrm{Fe}^{3+}$ and $\mathrm{Fe}^{4+}$, respectively. The $\mathrm{Fe}^{4+}$ ions provide the electron holes and control the conductivity, whereas $\mathrm{Fe}^{3+}$ controls via its spin structure and double exchange the magnetic properties. ${ }^{5}$

LSF10 was prepared by solid state synthesis and phase purity confirmed by x-ray diffraction (XRD).$^{1,6,7}$ The gravimetrically obtained $\delta$ is 0.01 . A single crystal (SC) was grown in an optical floating zone furnace and its dc conductivity determined by the four-probe technique. Laue XRD confirmed orthorhombic symmetry with space group Pbnm

\footnotetext{
a) Author to whom correspondence should be addressed. Empa-Swiss Federal Laboratories for Materials Testing \& Research, Überlandstrasse 129, CH8600 Dübendorf, Switzerland: Tel.: +41 (0)44 823 4850. FAX: +41 (0)44 823 4150. Electronic mail: artur.braun@alumni.ethz.ch.
}

62 and lattice constants $a=5.543 \AA, b=5.568 \AA$, and $c=7.846 \AA$ A. A $1 \mathrm{~mm}$ thick SC disk in [111] orientation was cut and high-quality surface finished. Part of the SC was pulverized and analyzed with XRD (wavelength of $1.936040 \AA$ ), at a heating rate of $25 \mathrm{~K} / \mathrm{min}$.

The $\mathrm{SC}$ was subject to $\mathrm{x}$-ray photoemission spectroscopy (PES) at $450 \mathrm{eV}$ excitation energy (Beamline 9.3.1, Advanced Light Source, Berkeley, California) after $\mathrm{Ar}^{+}$bombardment at $500 \mathrm{eV}$ for $30 \mathrm{~min}$, a heating cycle to $500 \mathrm{~K}$, and a scan for residual carbon. This procedure was repeated twice, and no carbon signal was detected. Temperature dependent $\mathrm{x}$-ray absorption spectra (XAS) of the $\mathrm{Fe} K$ edge were recorded at beamline BM29 of the European Synchrotron Radiation Facility, Grenoble, France.

LSF10 undergoes a transition from orthorhombic to rhombohedral at about $700 \mathrm{~K}^{1,2,6}$ The split of the (020) reflex at $41.5^{\circ}$ during temperature change suggests structural changes (Fig. 1). The slight shoulder at about $573 \mathrm{~K}$ around

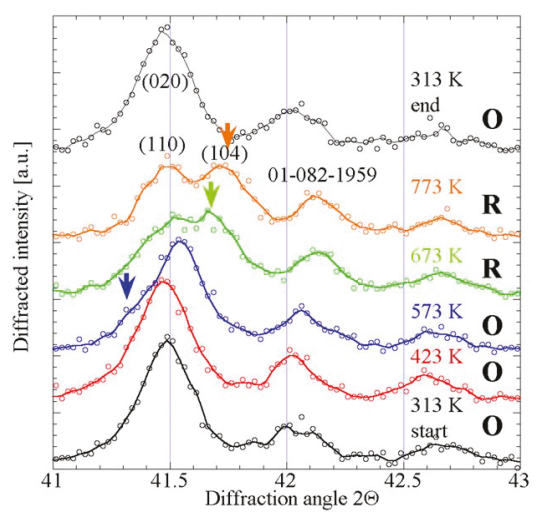

FIG. 1. (Color online) X-ray diffractogram of LSF10 powder from SC for $313<T<773 \mathrm{~K}$ in ambient atmosphere. 


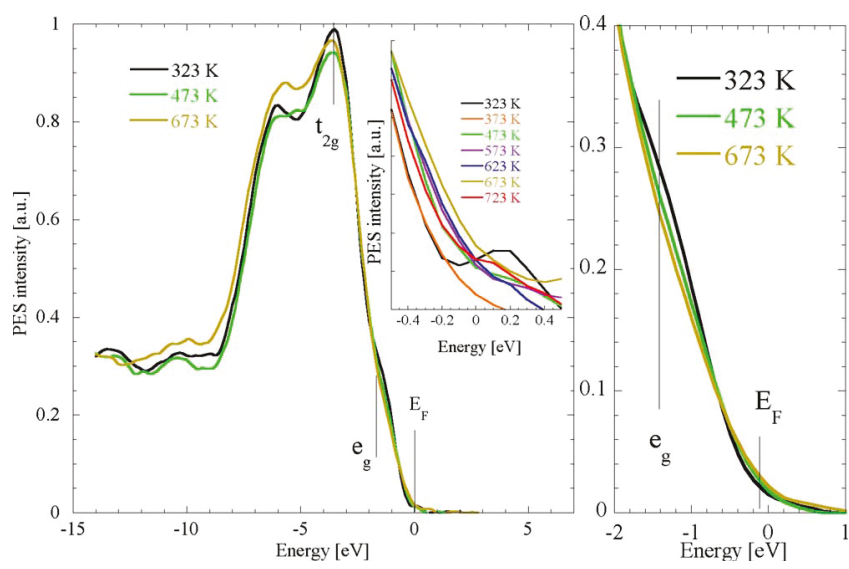

FIG. 2. (Color online) Valence band PES spectra of LSF10 SC for $323-723 \mathrm{~K}$. The inset in the left figure shows a magnification of the intensity near $E_{F}$. The right figure shows a magnification of the energy range of $-2<E<1 \mathrm{eV}$.

$41.35^{\circ}$ matches with tetragonal $\mathrm{Sr}_{3} \mathrm{Fe}_{2} \mathrm{O}_{7}$ (00-45-0398), possibly a transitional contamination, and becomes more pronounced with increasing $T$. At $773 \mathrm{~K}$, the reflex is split into peaks, separated by $0.2^{\circ}$. The general trend is that the diffracted intensity shifts towards larger angles, suggesting a decrease of the unit cell.

The assignment of the valence band features in the photoemission spectra (VB PES) in Fig. 2 is in accordance with Refs. 8-10. Upon heating, the intensity of the $t_{2 g}$ band $(-3.5 \mathrm{eV})$ and the $e_{g}$ band decrease, which is developed as a shoulder at about $-1.5 \mathrm{eV}$ below $E_{F}$. However, the intensity near $E_{F}$ increases slightly, i.e., intensity is redistributed towards $E_{F}$. The inset in Fig. 2 shows a magnification of the relevant range near $E_{F}$. First, the spectral weight at $373 \mathrm{~K}$ near $E_{F}$ is smaller than for the lower temperature of $323 \mathrm{~K}$. Second, the intensities for the following $T$ do increase, except for $T=723 \mathrm{~K}$, where the intensity is just below that of $473 \mathrm{~K}$, and above that of $323 \mathrm{~K}$. The spectrum at $673 \mathrm{~K}$ has thus the highest intensity near $E_{F}$. In order to quantify the intensity redistribution near $E_{F}$, we determined the intensity at $E_{F}$, and $100 \mathrm{meV}$ above and below $E_{F}$, and plotted these versus $T$ (Fig. 3, bottom). The three solid lines corresponding to the three energy positions show basically the same variation. Close inspection of the PES intensity variation curves reveals a correlation with features of the conductivity, which for comparison is plotted on the top in Fig. 3.

The increasing conductivity is typical for thermally activated small polaron hopping, where here with an activation energy of $E_{p}=317 \mathrm{meV} .{ }^{11}$ The conductivity attains its maximum at about $700 \mathrm{~K}$. On very close inspection, we notice two peculiarities: First is a reversible small jump at $357 \mathrm{~K}$ with a small hysteresis; the conductivity drops (jumps) upon heating (cooling) by about $0.025 \mathrm{~S} / \mathrm{cm}$. The inset in Fig. 3 (top) on the left magnifies the conductivity variation near this jump. The conductivity jump effect coincides with the valence band PES spectrum recorded at $373 \mathrm{~K}$ in Fig. 3, which shows a remarkable decrease in intensity near $E_{F}$.

The second peculiarity is a slight increase in the slope in the conductivity, i.e., an increase of $E_{p}$ to $332 \mathrm{meV}$ at about $573 \mathrm{~K}$. This observation is paralleled in the diffractogram at $573 \mathrm{~K}$ (Fig. 1), above which a clear transition from orthorhombic to rhombohedral is observed, suggesting that the rhombohedral structure is less conductive. Here we notice enhanced scattering of the conductivity, suggesting a struc-

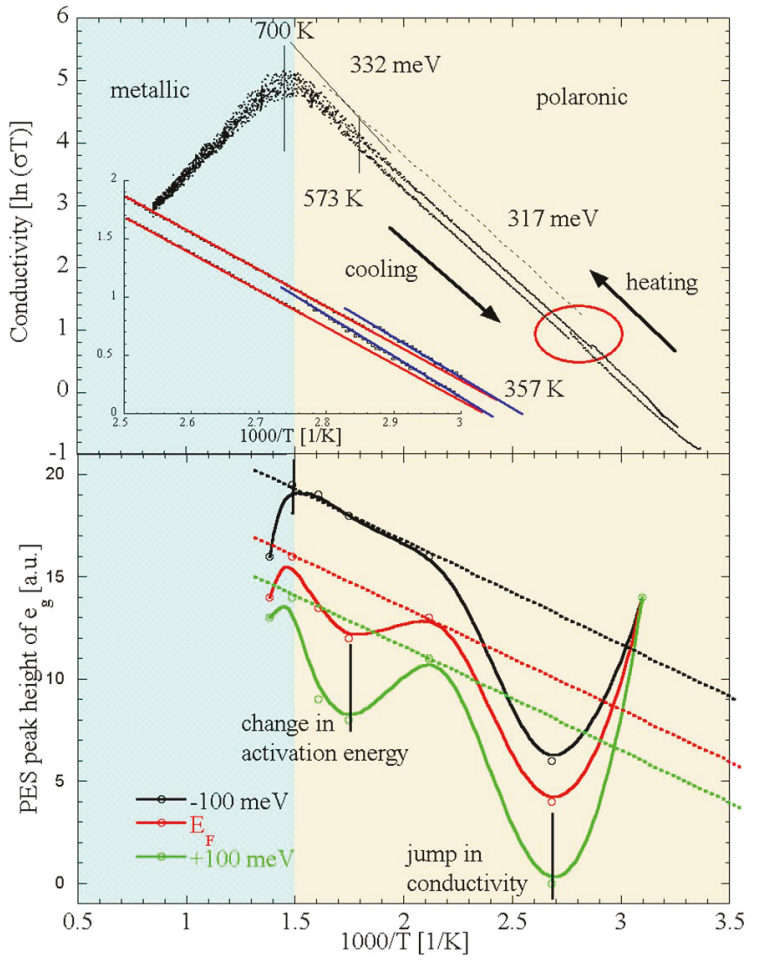

FIG. 3. (Color online) Variation of conductivity (top) and the conduction $e_{g}$ band height (bottom) as a function of temperature. Data are considered for $E_{F}$, and $100 \mathrm{meV}$ above and below $E_{F}$. The red circle region is magnified in the lower left part of the right figure and shows two tilted set (red for high, blue for low temperatures) of parallel lines.

turally poorly defined transitional stage. This behavior is to some extent reflected in the magnetic properties. We recollect that LSF is predominantly AFM, but may have canted ferromagnetic (FM) structure. The SC shows FM behavior at 300 and $400 \mathrm{~K}$, as evidenced by rectangular magnetization curves (not shown here). Out of the LSF series, LSF10 has the highest internal magnetic field at ambient $T$ and a Neél temperature of around $600 \mathrm{~K} .{ }^{1,12}$ The FM Curie point may be below $600 \mathrm{~K}$. Summarizing for $T \geqslant 573 \mathrm{~K}$, we believe that the structural transition increases the lattice distortion, impairing the $\mathrm{Fe}(3 d)-\mathrm{O}(2 p)$ hybridization via the doubleexchange angle $\theta$, and thus increasing the band width $W$ $\sim \cos (2 \theta)$ and $E_{g}{ }^{4}$. We speculate that at this temperature the FM Curie point is already passed, and that no doubleexchange-related conductivity decrease occurs.

The conjugated PES intensity increase near $E_{F}$ with increasing $T$ shows two extra features, having smaller spectral weight at $373 \mathrm{~K}$ than at $323 \mathrm{~K}$, and, subsequently, increasing spectral weight, with a maximum at about $700 \mathrm{~K}$, where $\sigma$ is maximal. The back-switching spectral weight at this temperature is hence paralleled by the maximum conductivity.

The $T$ dependent Fe $K$ edge XAS spectra (Fig. 4) point to the physical origin for the decrease of $\sigma$ and the cross-over in the conductivity mechanism above $700 \mathrm{~K}$. They show noticeable changes at 310,423 , and $573 \mathrm{~K}$. However, the spectra at 573 and $673 \mathrm{~K}$ differ insofar as the $673 \mathrm{~K}$ spectrum has a smaller chemical shift and hence represents a more reduced $\mathrm{Fe}$ species than those at lower $T$. With the exception for $773 \mathrm{~K}$, the white lines all coincide at $7127 \mathrm{eV}$. The $773 \mathrm{~K}$ spectrum has pronounced $e_{g}$ and $t_{2 g}$ prepeaks at 7104 and $7112 \mathrm{eV}$, indicative of $\mathrm{Fe}^{3+}$. This conclusion is supported by the position of the absorption edges at $7117.5 \mathrm{eV}$ for $773 \mathrm{~K}$ and $7121 \mathrm{eV}$ for $310 \mathrm{~K}$. The average nominal va- 


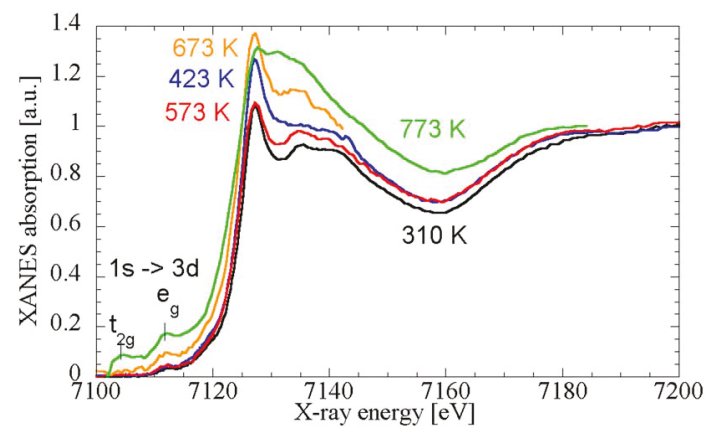

FIG. 4. (Color online) XAS of the Fe $K$ edge for $T=310-773 \mathrm{~K}$.

lence of $\mathrm{Fe}$ is 3.1 , brought about by $10 \% \mathrm{Fe}^{4+}$ and $90 \% \mathrm{Fe}^{3+}$. The chemical shift upon heating to $773 \mathrm{~K}$ is $3.5 \mathrm{eV}$, which accounts for about 0.75 valence formula units. ${ }^{13}$ Thus, the Fe is reduced from average $\mathrm{Fe}^{3.10}$ at $300 \mathrm{~K}$ to $\mathrm{Fe}^{2.35}$ at $773 \mathrm{~K}$. The XAS thus resemble to a large extent the $\mathrm{Fe}^{3+}$ features, as evidenced by the pre-edge peaks of $\mathrm{Fe}^{3+}$. The width of the white line at $773 \mathrm{~K}$ is larger than for lower $T$. White lines from ionic and covalent compounds are typically sharper than those from metals. This indicates that the ionic bond strength structure is softened during annealing and suggests a more metallic bonding, in line with the observed chemical shift towards lower energies. The decrease of the average iron valence is caused by oxygen loss during annealing, and, consequently, decreases the number of conducting holes. This manifests in decreasing conductivity and decline of the VB PES intensity at $T>700 \mathrm{~K}$.

This work is a high temperature application of VB PES on a rare-earth transition metal oxide perovskite. Ambient temperature studies on related materials were published recently. Matsuno et al. $^{14}$ found a decrease of spectral weight near $E_{F}$ towards $x=0.67$ (charge ordering state). $\mathrm{SrFeO}_{3}$ has the highest spectral weight at $E_{F}$, compared to LSF. Their studies $^{14}$ from 130 to $210 \mathrm{~K}$ indicated that changes are most pronounced for $x=0.67$ at $190 \mathrm{~K}$. Wadati et al. ${ }^{9}$ identified in the PES three main structures as from $\mathrm{Fe}(3 d)-\mathrm{O}(2 p)$ bonding states, and $t_{2 g}$ and $e_{g}$ states from Fe, the latter of which is next to the $E_{F}$. In the spectra at room temperature, the $e_{g}$ band next to $E_{F}$ became weaker but moved toward $E_{F}$ as $x$ increased towards better conducting compositions. They ${ }^{10}$ found for 10-260 K hole doping induced spectral weight transfer from below to above $E_{F}$ across the band gap in a highly non-rigidlike-band-manner. With increasing $T$, the weight of the $e_{g}$ band at $-1.5 \mathrm{eV}$ from $E_{F}$ decreased noticeably, with a corresponding weight increase at about $-0.2 \mathrm{eV}$ from $E_{F}{ }^{8}$

The three parallel dotted lines in the bottom of Fig. 3 reflect the global trend of the weight increase around $E_{F}$ with increasing $T$. The spectral weight maximum at about $700 \mathrm{~K}$ coincides with the conductivity maximum, and the two spectral weight minima coincide with the change of $E_{p}$ and the jump of $\sigma$ at about 573 and $357 \mathrm{~K}$, respectively. The intermediate PES maximum at about $463 \mathrm{~K}$ represents not a maximum that would correspond to a particular high conductivity, but the continuation of the overall linear increase of the spectral weight along with the temperature increases. It is noteworthy that the minute conductivity jump and the increase of $E_{a}$ cause significant changes in the trend of the spectral weight evolution during temperature change, whereas the global linear increase of the spectral weight is unspectacular. The overall linear behavior therefore accounts for the excitation of polarons as a primary response to thermal activation, while the distinct minima account for direct structural changes, which are secondary effects with respect to the temperature change. Comparison with calorimetry data confirms subtle yet systematic correlation between structural, spectral, and transport properties. The cause for the conductivity jump remains open.

The lightly $\mathrm{Sr}$ doped $\mathrm{La}_{1-x} \mathrm{Sr}_{x} \mathrm{FeO}_{3}$ has a thermally activated conductivity up to $673 \mathrm{~K}$ from polaron hopping of holes created by the $\mathrm{Fe}^{4+}$. The conductivity increase is reflected by the PES, i.e., spectral weight shift from the Fe $e_{g}$ band towards $E_{F}$. The density of states near $E_{F}$ is thus filled by the $\mathrm{Fe}^{4+}$ holes. Upon further heating, LSF10 adopts rhombohedral symmetry. Since $\mathrm{La}$ and $\mathrm{Sr}$ are virtually redox inert, the Fe valence adjusts to maintain charge balance, i.e., the $\mathrm{Fe}^{4+}$ is reduced, and the hole concentration decreases accordingly. This stage is met at about $700 \mathrm{~K}$, with maximum conductivity and its subsequent decrease. The temperature dependent XAS display significant changes, including the chemical shift towards smaller energies, confirming a reduction of the Fe. The PES spectrum at $723 \mathrm{~K}$ shows, consequently, a smaller spectral weight at $E_{F}$ than the other high temperature spectra. Since the attenuation length for $7210 \mathrm{eV}$ photons of around $3.5 \mu \mathrm{m}$ and the conductivity measurements are bulk representative, and the PES probing depth as applied here is below $20 \AA$, our study suggests that surface sensitive VB PES can be meaningful applied at high $T$ for LSF studies and permits correlation with bulk representative transport properties.

Funding by the E.U. MIRG No. CT-2006-042095, the Swiss NSF No. 200021-116688, CCMX NANCER and CCEM/OneBat. The ALS is supported by the Director, Office of Science/BES, of the U.S. DoE, No. DE-AC0205CH11231. ESRF and C. Prestipino are acknowledged for experiment No. HE2469.

${ }^{1}$ J.-C. Grenier, N. Ea, M. Pouchard, and M. M. Abou-Sekkina, Mater. Res. Bull. 19, 1301 (1984).

${ }^{2}$ S. E. Dunn, D. B. Currie, M. T. Weller, M. F. Thomas, and A. D. AlRawwas, J. Solid State Chem. 109, 134 (1994).

${ }^{3}$ D. D. Sarma, O. Rader, T. Kachel, A. Chainani, M. Mathew, K. Holldack, W. Gudat, and W. Eberhardt, Phys. Rev. B 49, 14238 (1994).

${ }^{4}$ S. K. Park, T. Ishikawa, Y. Tokura, J. Q. Li, and Y. Matsui, Phys. Rev. B 60, 10788 (1999)

${ }^{5}$ X.-D. Zhou, Q. Cai, J. Yang, M. Kim, W. B. Yelon, W. J. James, Y.-W. Shin, B. J. Scarfinio, and H. U. Anderson, J. Appl. Phys. 97, 10C314 (2005).

${ }^{6}$ A. Fossdal, M. Menon, I. Wærnhus, K. Wiik, M.-A. Einarsrud, and T. Grande, J. Am. Ceram. Soc. 87, 1952 (2004).

${ }^{7}$ A. Chainani, M. Mathew, and D. D. Sarma, Phys. Rev. B 48, 14818 (1993).

${ }^{8}$ H. Wadati, A. Chikamatsu, R. Hashimoto, M. Takizawa, H. Kumigashira, A. Fujimori, M. Oshima, M. Lippmaa, M. Kawasaki, and H. Koinuma, J. Phys. Soc. Jpn. 75, 054704 (2006).

${ }^{9}$ H. Wadati, D. Kobayashi, H. Kumigashira, K. Okazaki, T. Mizokawa, A. Fujimori, K. Horiba, M. Oshima, N. Hamada, M. Lippmaa, M. Kawasaki, and H. Koinuma, Phys. Rev. B 71, 035108 (2005).

${ }^{10}$ H. Wadati, D. Kobayashi, A. Chikamatsu, R. Hashimoto, M. Takizawa, K. Horiba, H. Kumigashira, T. Mizokawa, A. Fujimori, M. Oshima, M. Lippmaa, M. Kawasaki, and H. Koinuma, J. Electron Spectrosc. Relat. Phenom. 144, 877 (2005).

${ }^{11}$ W.-H. Jung, Physica B 299, 120 (2001).

${ }^{12}$ U. Shimony and J. M. Knudsen, Phys. Rev. 144, 361 (1966).

${ }^{13}$ A. J. Berry, H. St. C. O’Neill, K. D. Jayasuriya, S. J. Campbell, and G. J. Foran, Am. Mineral. 88, 967 (2003).

${ }^{14}$ J. Matsuno, T. Mizokawa, A. Fujimori, K. Mamiya, Y. Takeda, S. Kawasaki, and M. Takano, Phys. Rev. B 60, 4605 (1999). 